\title{
Classification of epileptic EEG signals based on simple random sampling and sequential feature selection
}

\author{
Hadi Ratham Al Ghayab - Yan Li $\cdot$ Shahab Abdulla • \\ Mohammed Diykh $\cdot$ Xiangkui Wan
}

Received: 4 December 2015 / Accepted: 3 February 2016/Published online: 27 February 2016

(C) The Author(s) 2016. This article is published with open access at Springerlink.com

\begin{abstract}
Electroencephalogram (EEG) signals are used broadly in the medical fields. The main applications of EEG signals are the diagnosis and treatment of diseases such as epilepsy, Alzheimer, sleep problems and so on. This paper presents a new method which extracts and selects features from multi-channel EEG signals. This research focuses on three main points. Firstly, simple random sampling (SRS) technique is used to extract features from the time domain of EEG signals. Secondly, the sequential feature selection (SFS) algorithm is applied to select the key features and to reduce the dimensionality of the data. Finally, the selected features are forwarded to a least square support vector machine (LS_SVM) classifier to classify the EEG signals. The LS_SVM classifier classified the features which are extracted and selected from the SRS and the SFS. The experimental results show that the method achieves $99.90,99.80$ and $100 \%$ for classification accuracy, sensitivity and specificity, respectively.
\end{abstract}

H. R. A. Ghayab $(\bowtie) \cdot$ Y. Li · S. Abdulla · M. Diykh

Faculty of Health, Engineering and Sciences, University of

Southern Queensland, Toowoomba, QLD 4350, Australia

e-mail: HadiRathamGhayab.AlGhayab@usq.edu.au

Y. Li

e-mail: Yan.Li@usq.edu.au

S. Abdulla

e-mail: Shahab.Abdulla@usq.edu.au

M. Diykh

e-mail: Mohammed.Diykh@usq.edu.au

X. Wan

School of Electrical and Electronic Engineering, Hubei

University of Technology, Wuhan 430068, China

e-mail: wanxiangkui@163.com
Keywords Electroencephalogram - Epileptic seizures · Simple random sampling - Sequential feature selection . Least square support vector machine

\section{Introduction}

Epilepsy is a disorder which affects the human brain and hugely impairs patients' daily lives. It is characterized by recurrent and sudden incidence of epileptic seizures [1]. According to an estimation of the World Health Organization, more than 50 million of population are affected by epilepsy [2,3]. Approximately, almost $1 \%$ population have the neurological disorders [4-6]. It leads to numerous research works to identify epilepsy and related treatments. Electroencephalogram (EEG) signals have been proved as a powerful tool for detecting and diagnosing different neurological diseases. EEG signals are often used to detect and classify epilepsy [7]. It is often difficult for the experts to recognize the people who have a brain disorder through visual inspection of EEG signals [8]. In addition, visual inspection for discriminating EEG signals is a time consuming, error prone, costly process and not sufficient enough for reliable information. The analysis and classification of EEG signals can lead to better diagnostic techniques for brain-related disorders. It is thus important to develop better EEG classification methods.

Many researchers developed new techniques to extract the significant information from EEG signals. The information is used as the input to different classifiers. There are many approaches used to extract the key features as well as to further select features. Most of these fall under five broad categories: time domain, frequency domain, timefrequency domain, traditional non-linear methods and graph theory approaches [9]. 
One of the methods used in this paper for extracting epileptic EEG data is sample random sampling (SRS) technique. Researchers often applied the SRS in time domain. In this technique, each sample of the population has the same chance to be selected as a subject. The complete process of sampling is done in a single step, with each subject can be selected independently from the other samples of the population [10]. Then, we forwarded all these samples to the sequential feature selection (SFS) method for selecting the best features.

This study uses the selected features as the input for a classifier. One of the most popular classifiers, the least square support vector machines (LS_SVMs) [11], is used to classify EEG data. This technique is used to identify the EEG data from healthy people and epileptic patients for epileptic seizures.

A lot of approaches for EEG signals classification have been developed [12]. There were reported a diverse of classification precisions for epileptic EEG data. Brief discussions of the previous research are provided below.

Gajic et al. [13] extracted different features from time, frequency, time-frequency domain and non-linear analysis.

These features were obtained from sub-bands with good representative characteristics. The researchers reduced the dimension of the features by using scatter matrices. This method yielded $98.7 \%$ accuracy.

An optimum allocation-based principal component analysis method was proposed by Siuly and Li [8] to extract key features for the classification of multi-class EEG signals from epileptic EEG data. They used four different classifiers which were LS_SVM, naive Bayes classifier, $k$-nearest neighbour (KNN) algorithm and linear discriminant analysis, to find out which one was the best classifier. They used four different output coding approaches for the multi-class LS_SVM. These were error correcting output codes, minimum output codes, one versus one (1vs1) and one versus all. That method achieved a $100 \%$ accuracy with LS_SVM_1vs1.

Feature extraction was carried out through an empirical mode decomposition. The extracted features were forwarded to two classifiers, the classification and regression tree and the $\mathrm{C} 4.5$ classifiers. The method using the $\mathrm{C} 4.5$ classifier suggested by Martis et al. [14] obtained good experimental results of 95.33, 98 and $97 \%$ for accuracy, sensitivity and specificity, respectively.

Chua et al. [15] gained features from raw EEG recordings by using higher order spectra. They used a Gaussian mixture model (GMM) and a SVM classifiers to detect epileptic EEG signals. They achieved average accuracies of 93.11 and $92.56 \%$ for the HOS based GMM classifier and the SVM classifier, respectively, for different EEG classes, such as normal, pre-ictal and epileptic EEGs.

On the other hand, a genetic algorithm (GA) was used by Guo et al. [16] to automatically extract features from EEG data in order to enhance the classifier's performance, as well as, to reduce the feature's dimensionality. They used two groups of epileptic datasets. The first group was two classes of healthy people and epileptic patients. The second group was three classes of healthy people, inter-ictal and ictal. The KNN classifier was used in the work to classify the two groups. They gained 88.6 and $99.2 \%$ accuracies for the first group without GA and with GA, respectively. They obtained of a $67.2 \%$ accuracy without GA, and $93.5 \%$ within GA, respectively, for the second group.

Ocak decomposed EEG signals, which were recorded from normal subjects and epileptic patients, by using discrete wavelet transform [17]. An approximate entropy (ApEn) was extracted from the approximation and the detail coefficients. The methodology achieved more than $96 \%$ accuracy.

Srinivasan et al. used the ApEn to extract features and an artificial neural network classifier to identify epileptic EEG signals [18]. That approach achieved a high overall accuracy of $100 \%$.

Srinivasan et al. also proposed a special type of recurrent neural network, Elman network [19]. They used the feature extracted in time domain and frequency domain as the input to the proposed classifier. The Elman network method yielded a $99.6 \%$ accuracy with a single input feature.

A wavelet transform method was used by Gajic et al. [20] to extract the key features. They also used scatter matrices to reduce the dimensionality of the features. These features were used as the input to a quadratic classifier. The EEG epileptic database was classified into healthy subjects, epileptic subjects during a seizure-free (inter-ictal) and epileptic patients during the seizure activity (ictal). They obtained a $99 \%$ classification accuracy.

Shen et al. [12] proposed a cascade of wavelet-ApEn for feature selection. They used Fisher scores for adaptive feature selection, and SVM for feature classification to detect epileptic seizures. They applied the method to different epileptic EEG recordings: open source EEG data and clinical EEG data. The method obtained the overall classification accuracies of 99.97 and $98.73 \%$, respectively.

A sampling technique (ST) based on a LS_SVM was proposed by Siuly et al. [21]. Firstly, they used the ST to extract features from two classes of, normal persons with eyes open and epileptic patients during a seizure activity. They applied the LS_SVM to the extracted features. The total classification accuracy by that approach for both the training and testing datasets was 80.31 and $80.05 \%$, respectively.

Husain and Rao [22] presented an artificial neural network model using back propagation algorithm for the classification of epileptic EEG signals. They decomposed the EEG signals into a finite set of band limited signals termed as intrinsic mode functions. They also applied 
Hilbert transform on these intrinsic mode functions to calculate instantaneous frequencies. They achieved a $99.80 \%$ overall classification accuracy.

Rückstieß et al. [23] performed a SFS method to select the most representative features at each time step. Each successive features depended on the previous features. All the features were put into one vector and were forwarded to a classifier. This approach was applied for handwritten digits classification and a medical diabetes prediction task.

A sequential floating forward selection (SFFS) algorithm was proposed to detect epileptic seizures in EEG signals by Choi et al. [24]. They selected the most energy power as the features from frequency bands by using the SFFS algorithm. The total accuracy obtained by that method was $97.2 \%$.

In this study, we developed a new method combining the SRS with the SFS to acquire the best features set, and then we use the features as the input of the LS_SVM classifier for the EEG classification. All the techniques are discussed in Sects. 3 and 4. The conclusion is presented in Sect. 5.

\section{Experimental data}

The data used in this study are open source EEG recordings and are publicly available ${ }^{1}$ [25]. The database includes five sets of EEG recordings (sets $\mathbf{A}-\mathbf{E}$ ), with each containing 100 single-channel EEG signals of $23.6 \mathrm{~s}$ from five separate classes. References $[13,26]$ presented all details of these datasets from set A to E. This study selected set A which was taken from surface EEG recordings of five healthy people with eye open, and set $\mathbf{E}$ which was taken from EEG records of five pre-surgical epileptic patients during epileptic seizure activity.

\section{Methodology}

The big EEG datasets cause the curse of dimensionality and make it difficult to estimate the accuracy of classification from a limited number of samples. This study develops a new structure for classifying epileptic EEG signals, as presented in Fig. 1. This work investigates and explores whether the SRS combined with SFS give the best features for epileptic EEG signals classification.

\subsection{Simple random sampling (SRS) technique}

SRS technique is a popular type of random or prospect sampling [21]. In this technique, each sample of the population has the same chance of being selected as a subject.

\footnotetext{
$\overline{1} \mathrm{http} / / / \mathrm{www} . \mathrm{meb} . u n i b o n n . d e /$ epileptologie/science/physik/eegdata. html.
}

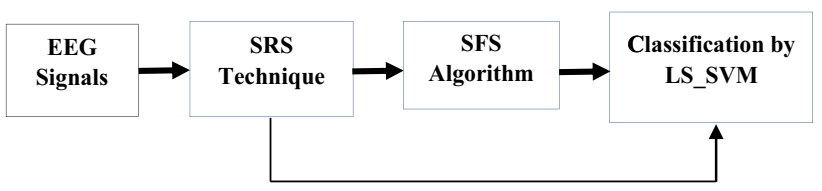

Fig. 1 The structure of the proposed system

We put the number of population in a sample size calculator of the "Creative Research System" (available in sample size calculator online), to determine the sample size for both samples and subsamples. In this work, the dataset used are set $\mathbf{A}$ and set $\mathbf{E}$ (repeated). Each set has 100 data files, and each file has 4097 observations.

This research uses the sample size calculator to find the sample size needed as well as to find the subsample size. The sizes of the samples and the subsamples in this work are 3288 and 2746, respectively. The sizes were selected because they reflect the limitation of time to select samples and subsamples. Firstly, we randomly select 10 samples from size 3288 for each dataset (set A or E). Secondly, 5 subsamples are also random chosen from each 10 random samples, with a size of 2746 . In each step, this study takes into account a 99-100\% confidence interval and a $99 \%$ confidence level. In the last step, nine statistical features are extracted from each subsample. These features are \{maximum value (Max), minimum value (Min), mean value, median value, mode, first quartile (Xq1), second quartile $(\mathrm{Xq} 2)$, range value and standard deviation $(\mathrm{Std})$. Figure 2 shows how samples, subsamples and features are taken from each class. We used MATLAB software package version 8.4 , R2014b, for the experiments.

\subsection{Sequential feature selection (SFS) algorithms}

The SFS is used to reduce the dimensionality of the dataset selected randomly from the SRS. This method is used to generate fewer numbers of uncorrelated variables which are utilized as the features for the better classification of EEG signals. The aim of the presented sequential selection algorithm is to decrease the feature space, $\mathbf{D}=x_{1}, x_{2}, \ldots, x_{n}$, to a subset of features, $\mathbf{D}-n$. It aims at enhancing or optimizing the computational execution of the classifier, as well as avoiding the curse of dimensionality [27]. This method is used to select a sufficiently reduced subset from the feature space $\mathbf{D}$ without affecting the performance of the classifier. In order to choose a suitable feature subset size $k$, namely, a criterion function typically estimates the recognition rate of the classifier [28]. The SFS algorithm starts with an empty set $\boldsymbol{S}$, and progressively fills the set $\boldsymbol{S}$ through adding features selected by the criterion function $[29,30]$. It is searching on the feature space from bottom to up. Figure 3 illustrates how the SFS picks features from the original data. The SFS is applied to select the best features 
Fig. 2 The SRS technique to select samples, subsamples and statistical features

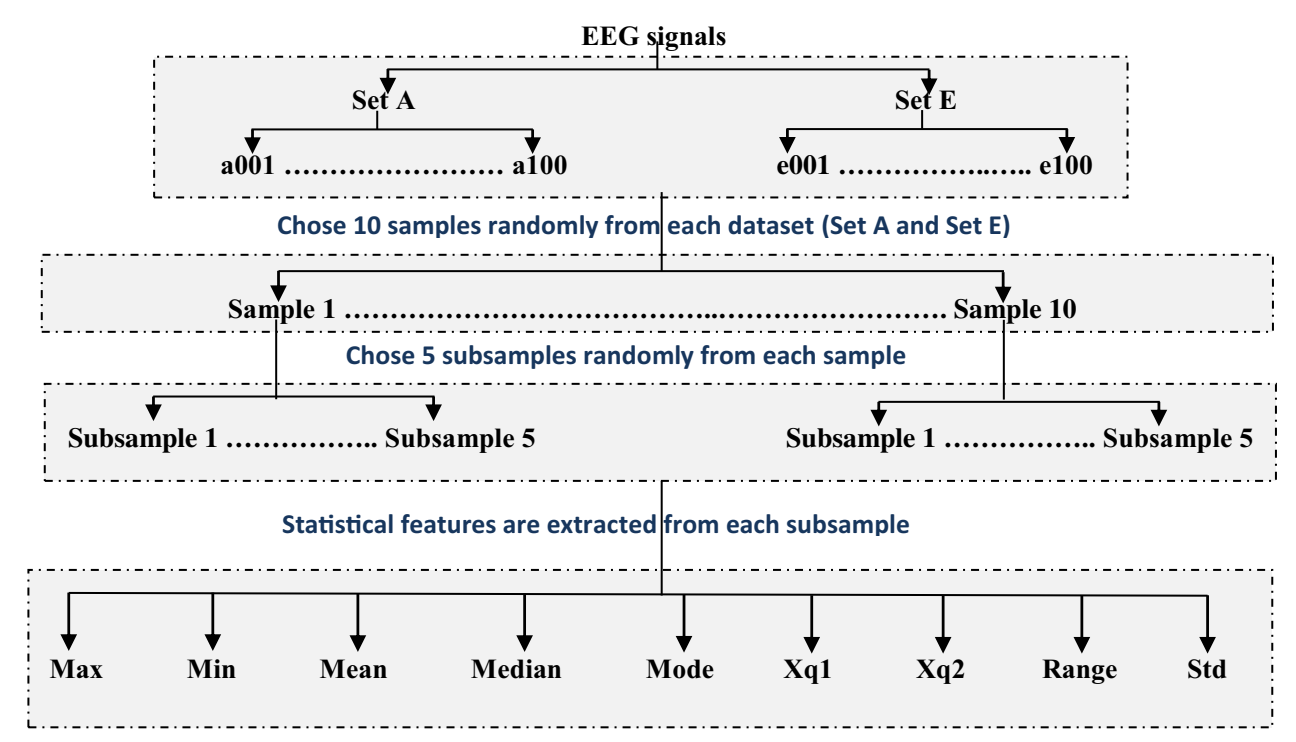

EEG signals

Chose 10 samples randomly from each dataset (Set A and Set E)

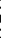

\section{(1)} $\vdots$

Statistical features are extracted from each subsample

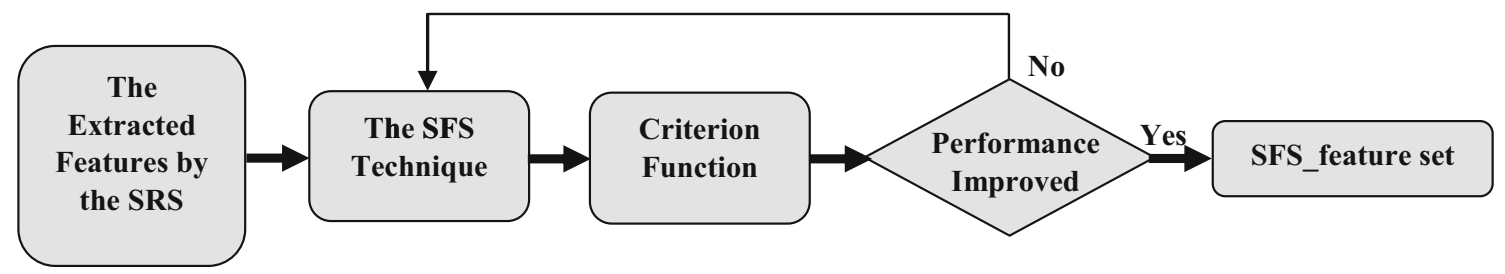

Fig. 3 Features selection from the extracted features by the SRS

from the statistical features. The criterion is empirically chosen based on the experimental results. In this study, several experiments are made to define the best criterion. The criterion value is calculated based on the statistical relations among the features. Firstly, the Max value is chosen as the criterion as shown in Eq. (1).

$\delta=\rho \sum_{i=1}^{n} f s 2(i) \quad i=1,2, \ldots, n$,

where $\delta$ refers to the criterion, $\rho$ is one of the nine statistical features, $n$ is the number of the features and fs 2 is the statistical feature set. Secondly, all the features are selected in the same way for Min, Mean, Mode and Std, in order to find the best features by the SFS algorithm. The best features (denoted as SFS_feature) are selected based on Eqs. (2) and (3) as below:

$$
\begin{aligned}
& \delta \leq f s 2 \\
& \delta>f s 2 .
\end{aligned}
$$

\subsection{The feature set}

After decreasing the dimensions of the features through the SFS, the new feature set is forwarded to the LS_SVM classifier. In this study, we obtain a feature set that has 2000 data points of 35 dimensions. These features are divided into two groups, which are the training set and the testing set. The training set is directed to train a classifier. The testing set is employed to evaluate the performance of the methodology and it is utilized as the input of the classifier.

\subsection{Least square support vector machines}

In this subsection, we briefly review some basic work on LS_SVMs for classification. LS_SVMs are proposed by Suykens and Vandewalle. LS_SVMs are the least square versions of SVMs, which are a set of related supervised learning methods that analyse data and recognize patterns. Moreover, they are used for classification and regression analysis [31]. In this research, the LS_SVM classifier with a radial basis function kernel is used for the classification of epileptic EEG signals. These classifiers can avoid the problem of convex quadratic programming from the classical SVMs by using a set of linear equations [8]. In this paper, the classification is performed by LS_SVMlab (version 1.8) toolbox in MATLAB ${ }^{2}$ [32].

\footnotetext{
${ }^{2}$ http://www.esat.kuleuven.ac.be/sista/lssvmlab/.
} 


\subsection{Performance measures}

This subsection presents assessing how the proposed method performs. The assessments include accuracy (also known as recognition rate), sensitivity (or recall) and specificity. The accuracy of a classifier is the percentage of the test set which is correctly classified by the classifier. The sensitivity is referred to the true positive rate which is the proportion of the positive set correctly identified.

The specificity is the true negative average which is the proportion of the negative set correctly identified. The following Eqs. (4)-(6) provide the definitions for the terms [33]:

Accuracy $=\frac{\mathrm{TP}+\mathrm{TN}}{\mathrm{P}+\mathrm{N}}$,

Sensitivity $=\frac{T P}{P}$,

Specificity $=\frac{\mathrm{TN}}{\mathrm{N}}$,

where TP is the number of true positives, TN is the number of true negatives and $\mathrm{P}$ and $\mathrm{N}$ are the positive and negative samples, respectively.

\section{Results and discussions}

In this study, we involved two datasets: sets $\mathbf{A}$ and $\mathbf{E}$ as mentioned in Sect. 2. SRS technique was used to extract features from the datasets. This technique selected features randomly by choosing 10 samples from each dataset (sets $\mathbf{A}$ and $\mathbf{E}$ ). A five subsamples were selected from each sample. From each subsample, nine statistical features,

Table 1 Classification accuracy for epileptic EEG signals (sets $\mathbf{A}$ and $\mathbf{E}$ )

\begin{tabular}{ll}
\hline Statistical parameters & Results $(\%)$ \\
\hline Accuracy & 100 \\
Sensitivity & 100 \\
Specificity & 100 \\
\hline
\end{tabular}

such as minimum, maximum, mean, median, mode, first quartile, third quartile, inter-quartile range and Std were extracted as aforementioned in Sect. 3.1.

A set of features obtained from the SRS included $2000 \times 45$ dimensions. These features were used in two different ways. Firstly, the statistical features were directly fed to the LS_SVM classifier and yielded the results, as shown in Table 1. Secondly, the SFS based on the criterion was employed to select the key features from the extracted features as mentioned in Sect. 3.2. As shown in the results, the good results of the best features are presented in Table 2. In Table 2, the good results are obtained by using the SRS algorithm and the SFS technique with the LS_SVM classifier depending on the best criterion chosen. Furthermore, the LS_SVM has two important parameters, which are $\gamma$ and $\sigma^{2}$ which should be suitably selected for achieving a desirable performance too. The LS_SVM was affected by the value of these two parameters. This study trained the LS_SVM with different groups of the parameters $\gamma$ and $\sigma^{2}$ to obtain best results. In this proposed method, we conducted with one group of the five EEG datasets and gained the best classification result with sets $\mathbf{A}$ and $\mathbf{E}$ when $\gamma=10$ and $\sigma^{2}=1$ for the two methods applied in this paper. The results of the proposed method were compared with the results that were obtained from the SRS method and the LS_SVM classifier. The experimental results showed that our approach yielded $99.90 \%$ classification accuracy for the epileptic EEG data. Table 3 gives a better view for the results by the two different classification methods. On the other hand, in this study, the evaluation of time complexity between the presented approach and the SRS was conducted.

The SRS_SFS_LS_SVM method took $0.16 \mathrm{~s}$ to classify the extracted features in Sect. 3.2. While the SRS_LS_SVM tackled the same features with $1.52 \mathrm{~s}$ as shown in Table 3. The performance of the proposed method is also compared with two existing methods in the literature. For fair comparison, the same dataset was used in comparison. The results show that the proposed method outperforms over the other two existing methods: a HuangHilbert transform and an artificial neural network model by Husain and Rao [22] and a ST and LS_SVM methods by
Table 2 Experimental results using different statistic features as the criterion

\begin{tabular}{llcc}
\hline Choose criterion & Accuracy $(\%)$ & Sensitivity $(\%)$ & Specificity $(\%)$ \\
\hline Mean $\geq$ fs2 (SFS_feature) & 99.90 & 99.80 & 100.00 \\
Mean $\leq$ fs2 (SFS_feature) & 98.90 & 98.00 & 99.80 \\
Max $\leq$ fs2 (SFS_feature) & 97.20 & 100.00 & 94.40 \\
Min $\geq$ fs2 (SFS_feature) & 99.10 & 99.20 & 99.00 \\
Mode $\geq$ fs2 (SFS_feature) & 97.70 & 95.40 & 100.00 \\
Median $\leq$ fs2 (SFS_feature) & 95.30 & 92.80 & 97.80 \\
Std $\geq$ fs2 (SFS_feature) & 95.60 & 91.20 & 100.00 \\
\hline
\end{tabular}


Table 3 Comparison of the results and time complexity for the proposed method with other methods

\begin{tabular}{lcrcc}
\hline Methods & Accuracy $(\%)$ & Sensitivity $(\%)$ & Specificity $(\%)$ & Time (s) \\
\hline SRS_LS_SVM & 100.00 & 100.00 & 100.00 \\
The proposed method with the best criterion (SRS_SFS_LS_SVM) & 99.90 & 99.80 & 1.52 \\
\hline
\end{tabular}

Table 4 Comparison of performance of our proposed method with two recently reported methods for sets $\mathbf{A}$ and $\mathbf{E}$ of the EEG epileptic database

\begin{tabular}{llll}
\hline Different methods & Accuracy (\%) & Sensitivity (\%) & Specificity (\%) \\
\hline The proposed method with the best criterion (SRS_SFS_LS_SVM) & 99.90 & 99.80 & 100 \\
A Huang-Hilbert transform and an artificial neural network model [22] & 99.80 & 99.75 & 100 \\
A sampling technique and LS_SVM method [21] & 80.05 & 74.97 & 87.70 \\
\hline
\end{tabular}

Siuly et al. [21]. The performance comparison of the proposed method with the two reported methods to classify sets $\mathbf{A}$ and $\mathbf{E}$ is shown in Table 4. Husain and Rao in 2014 applied a Huang-Hilbert transform and an artificial neural network model on sets $\mathbf{A}$ and $\mathbf{E}$ (the same datasets used in this paper). They achieved a $99.80 \%$ classification accuracy. While Siuly et al. in 2009 obtained $80.05 \%$ classification accuracy when they used a ST and the LS_SVM methods to classify the EEG signals for the same datasets. Moreover, the proposed method gains a $99.90 \%$ classification accuracy for the same group of datasets. The results shown that the proposed technique in this paper has the potential to classify the EEG signals from healthy people and epileptic patients using the extracted and selected features from the SRS and SFS techniques.

\section{Conclusions}

This research concentrates on two classes of EEG signals from healthy people and epileptic patients. The study presents a SRS_SFS method to extract and select the key features for classifying EEG signals into two classes. The LS_SVM classifier is used to classify two-category EEG data after the feature extraction and selection. This method yields the results of $99.90,99.80$ and $100 \%$ for classification accuracy, sensitivity and specificity, respectively. In addition, the proposed method is faster than the SRS technique. It means that the SRS_SFS is useful for extracting and selecting the EEG features. To sum up, the proposed method is very efficient for analysing and classifying epileptic EEG signals. It will be also useful for the classification of other biomedical data.

Open Access This article is distributed under the terms of the Creative Commons Attribution 4.0 International License (http://crea tivecommons.org/licenses/by/4.0/), which permits unrestricted use, distribution, and reproduction in any medium, provided you give appropriate credit to the original author(s) and the source, provide a link to the Creative Commons license, and indicate if changes were made.

\section{References}

1. Buck D, Baker GA, Jacoby A et al (1997) Patients' experiences of injury as a result of epilepsy. Epilepsia 38(4):439-444

2. World Health Organization (WHO) (2011). Report: WHO. http:// www.who.int/mediacentre/factssheets/fs999/en/index.html. Accessed Dec 2015

3. Mcgrogan N (1999) Neural network detection of epileptic seizures in the electroencephalogram. http://www.new.ox.ac.uk/ $\sim$ nmcgroga/work/transfer

4. Boer H, Engel J, Prilipko L (2005) Global campaign against epilepsy. Epilepsy Atlas 82-83

5. Iasemidis LD (2003) Epileptic seizure prediction and control. IEEE Trans Biomed Eng 50(5):549-558

6. Kumar TS, Kanhangad V, Pachori RB (2015) Classification of seizure and seizure-free EEG signals using local binary patterns. Biomed Signal Process Control 15:33-40

7. Adeli H, Zhou Z, Dadmehr N (2003) Analysis of EEG records in an epileptic patient using wavelet transform. J Neurosci Methods 123(1):69-87

8. Siuly S, Li Y (2015) Designing a robust feature extraction method based on optimum allocation and principal component analysis for epileptic EEG signal classification. Comput Methods Programs Biomed 119(1):29-42

9. Acharya UR, Sree SV, Swapna G et al (2013) Automated EEG analysis of epilepsy: a review. Knowl Based Syst 45:147-165

10. Barreiro PL, Albandoz JP (2001) Population and sample. Sampling techniques. Management mathematics for European schools, MaMaEusch (994342-CP-1-2001-1-DECOMENIUSC21)

11. Wu F, Zhao Y (2005) Least squares support vector machine on Moret wavelet kernel function. In: International conference on neural networks and brain, 2005. ICNN\&B'05. IEEE, Beijing, p 327-333

12. Shen C-P, Chen C-C, Hsieh S-L et al (2013) High-performance seizure detection system using a wavelet-approximate entropyfSVM cascade with clinical validation. Clin EEG Neurosci $44: 247-256$ 
13. Gajic D, Djurovic Z, Gligorijevic J et al (2015) Detection of epileptiform activity in EEG signals based on time-frequency and non-linear analysis. Front Comput Neurosci. doi:10.3389/ fncom.2015.00038

14. Martis RJ, Acharya UR, Tan JH et al (2012) Application of empirical mode decomposition (EMD) for automated detection of epilepsy using EEG signals. Int J Neural Syst 22(6):1250027

15. Chua KC, Chandran V, Acharya UR et al (2011) Application of higher order spectra to identify epileptic EEG. J Med Syst 35(6): 1563-1571

16. Guo L, Rivero D, Dorado J et al (2011) Automatic feature extraction using genetic programming: an application to epileptic EEG classification. Expert Syst Appl 38(8):10425-10436

17. Ocak H (2009) Automatic detection of epileptic seizures in EEG using discrete wavelet transform and approximate entropy. Expert Syst Appl 36(2):2027-2036

18. Srinivasan V, Eswaran C, Sriraam N (2007) Approximate entropy-based epileptic EEG detection using artificial neural networks. IEEE Trans Inf Technol Biomed 11(3):288-295

19. Srinivasan V, Eswaran C, Sriraam N (2005) Artificial neural network based epileptic detection using time-domain and frequency-domain features. J Med Syst 29(6):647-660

20. Gajic D, Djurovic Z, Di Gennaro S et al (2014) Classification of EEG signals for detection of epileptic seizures based on wavelets and statistical pattern recognition. Biomed Eng Appl Basis Commun 26:1450021

21. Siuly S, Li Y, Wen P (2009) Classification of EEG signals using sampling techniques and least square support vector machines. In: Rough sets and knowledge technology. Springer, Berlin, pp 375-382

22. Husain SJ, Rao K (2014) An artificial neural network model for classification of epileptic seizures using Huang-Hilbert transform. Int J Soft Comput 5(3):23

23. Rückstieß T, Osendorfer C, Van Der Smagt P (2011) Sequential feature selection for classification. In: AI 2011: advances in artificial intelligence. Springer, Berlin, pp 132-141

24. Choi K-S, Zeng Y, Qin J (2012) Using sequential floating forward selection algorithm to detect epileptic seizure in EEG signals. In: 2012 IEEE 11th international conference on signal processing (ICSP). IEEE, Beijing, pp 1637-1640

25. EEG time series (Nov 2005). http://www.meb.unibonn.de/epi leptologie/science/physik/eegdata.html. Accessed Nov 2015

26. Andrzejak RG, Lehnertz K, Mormann F et al (2001) Indications of nonlinear deterministic and finite-dimensional structures in time series of brain electrical activity: dependence on recording region and brain state. Phys Rev E 64:061907

27. Marcano-Cedeño A, Quintanilla-Domínguez J, Cortina-Januchs $M$ et al (2010) Feature selection using sequential forward selection and classification applying artificial metaplasticity neural network. In: 36th annual conference on IEEE Industrial Electronics Society. USA, pp 2845-2850

28. Ferri F, Pudil P, Hatef M et al (1994) Comparative study of techniques for large-scale feature selection. Pattern Recognit Pract IV:403-413
29. Pudil P, Novovičová J, Kittler J (1994) Floating search methods in feature selection. Pattern Recognit Lett 15(11):1119-1125

30. Reunanen J (2003) Overfitting in making comparisons between variable selection methods. J Mach Learn Res 3:1371-1382

31. Suykens JA, Vandewalle J (1999) Least squares support vector machine classifiers. Neural Process Lett 9(3):293-300

32. LS-SVMlab toolbox (version 1.8). http://www.esat.kuleuven.ac. be/sista/lssvmlab/. Accessed Nov 2015

33. Han J, Kamber M, Pei J (2011) Data mining: concepts and techniques, 3rd edn. Elsevier, Amsterdam

Hadi Ratham Al Ghayab received his BSc Degree in Computer Science from Thi_Qar University in 2007 and MSc Degree in Information Technology from University Utara Malaysia in 2010. He is currently a PhD Student in the Faculty of Health, Engineering and Sciences, University of Southern Queensland Toowoomba. His research interests include biomedical signal analysis, data mining and image processing.

Yan $\mathbf{L i}$ is currently an Associate Professor of Computer Sciences in the Faculty of Health, Engineering and Sciences at the University of Southern Queensland, Australia. Her research interests are in the areas of Big Data Technologies, Artificial Intelligence, Biomedical Engineering, and Signal/Image Processing.

Shahab Abdulla received his BSc and MSc Degrees from University of Technology Baghdad and PhD from USQ. He is currently a Lecturer in Language Centre, University of Southern Queensland. His research interests are in the areas of biomedical engineering, complex medical engineering, networked system, intelligent control, computer control systems, robotics and mathematics research, etc.

Mohammed Diykh received his BSc Degree in Computer Science from Thi_Qar University and MSc Degree in Information Technology from Voronezh State University in 2002 and 2010, respectively. He is currently a PhD Student in the Faculty of Health, Engineering and Sciences, University of Southern Queensland Toowoomba. His research interests include biomedical signal analysis, data mining and image processing, etc.

Xiangkui Wan is a Professor of the school of Electrical and Electronic Engineering, Hubei University of Technology. He received MS Degree and PhD Degree in Mechanical and Electronic Engineering from the Chongqing University, in 2002 and 2005, respectively. From 2005 to 2008, he was with the Department of Information Engineering, Guangdong University of Technology, as a Lecturer. Since 2008, he was as an Associate Professor in the same Department. And since 2014, he is with the School of Electrical and Electronic Engineering, Hubei University of Technology. His professional research interests are in signal processing of biomedical signals, artifact and noise analysis and linear and nonlinear time-series analysis. 